\title{
Duration of Hospital Stay in Alcohol/Substance Addictions and Psychiatric Disorders: A 12-Year Retrospective Analysis
}

\author{
Bahadir Geniş ${ }^{1 *}$, Behcet Cosar $^{2}$ \\ 1Department of Psychiatry, Caycuma State Hospital, Zonguldak, Turkey \\ ${ }^{2}$ Department of Psychiatry, Faculty of Medicine, Gazi University, Ankara, Turkey \\ *Corresponding Author: Bahadır Geniş, MD-Specialist, Department of Psychiatry, Caycuma State Hospital, Caycuma, \\ Zonguldak, Turkey. Tel: +90-372 615 8222, Email: bahadirgenis06@gmail.com
}

Received October 21, 2019; Accepted February 12, 2020; Online Published February 29, 2020

\begin{abstract}
Background: Mental disorders are generally a significant reason for increased morbidity. They constitute a serious disease burden. One of the main reasons for this disease burden is long hospitalization periods.

Objective: The current study investigated the length of hospital stay and the variables affecting it in patients treated in the Gazi University Hospital Psychiatry Department between 2005-2016.

Methods: Patient diagnoses were analyzed according to the International Classification of Diseases 10th Revision (ICD-10). Data was obtained for 7027 hospitalizations over a 12-year period. Records of repeated hospitalizations, non-psychiatric primary diagnoses, and missing data were not included in the analysis. As a result, data from 5129 hospitalizations were included in the analysis.

Results: Mean age of the sample was $45.27 \pm 14.69$, and $62.5 \%(n=3204)$ of the patients were male. Mean hospitalization period was $28.66 \pm 17.25$ days. Schizophrenia and depressive disorder significantly prolonged hospital stay, while substance addiction shortened the duration of hospitalization $(P<0.001)$. It was found that the duration of hospitalization decreased significantly over the years $(P<0.001)$. Advanced age $(P<0.001)$, recurrent admission $(P<0.001)$, and female gender $(P=0.029)$ were other variables affecting this period.

Conclusion: Schizophrenia and depression are the most common psychiatric disorders in the inpatient service, and these disorders prolong hospitalization periods. The duration of hospital stay is considerably less in substance addiction than in other psychiatric disorders. Non-clinical variables, such as year of hospitalization, may affect the length of hospital stay. Keywords: Hospitalization, Psychiatric Disorders, Alcohol Addiction, Substance Addiction, Length of Stay, Schizophrenia
\end{abstract}

\section{Background}

Mental health is defined by the World Health Organization (WHO) as "a state of well-being in which every individual realizes his or her own potential, can cope with the normal stresses of life, can work productively and fruitfully, and is able to make a contribution to her or his community." ${ }^{1}$ Moreover, it is known that mental health is disrupted at any stage of life in one in every four people or at an even higher rate. ${ }^{2}$

In general, mental disorders are significant reasons for increased morbidity and disability. ${ }^{3,4}$ There are five mental disorders listed in the first twenty diseases of the Global Burden of Disease (GBD) report. ${ }^{3}$ Among these, major depression is ranked in second, anxiety disorders in seventh, schizophrenia in eleventh, dysthymia in sixteenth, and bipolar disorder in seventeenth place. Some of the most significant reasons why these disorders have a high disease burden are their recurring character, the insufficiency of existing treatments, their effects on families, nonconforming health policies, and stigmatization. ${ }^{4}$ One main reason for the disease burden caused by psychiatric disorders is long hospitalization periods. ${ }^{5}$ Numerous studies have been carried out both for decreasing the cost imposed by hospital stays and to understand the nature of diseases. It has been determined that a high number of variables associated with psychiatric disorders, such as treatment modality, gender, age, diagnosis, and the presence of physical comorbidities, affect the duration of hospitalization..$^{5-7}$

The WHO 2018 drug report revealed that 275 million people (5.6\%) aged 15-64 used drugs at least once a year. ${ }^{8}$ Approximately 31 million people who use drugs have a drug use disorder. WHO's 2018 alcohol report stated that 3.1 billion people are alcohol users and the rate of

Copyright (C) 2020 The Author(s). This is an open-access article distributed under the terms of the Creative Commons Attribution License (http:// creativecommons.org/licenses/by/4.0), which permits unrestricted use, distribution, and reproduction in any medium, provided the original work is properly cited. 
alcohol use disorder is $5.1 \% .{ }^{9}$ These alcohol and substance addictions place a serious burden on the global economy. ${ }^{8,9}$ One of these burdens is the length of hospitalization which is the topic of different debates. In addictions, there are important problems such as recurrent and chronic features of the disorders, accompanying psychiatric disorders, and treatment incompatibilities in patients. ${ }^{10,11}$ These problems affect the treatment process and change the duration of the hospitalization period. ${ }^{12,13}$ Addictions are accompanied by many psychiatric disorders, primarily depression. ${ }^{13}$ In cases of addiction and psychiatric comorbidities, which one is the cause and which one is the result is not generally clear. ${ }^{13}$ However, it is known that addictions increase the severity of psychiatric disorders, decrease the response to treatment, and prolong treatment periods. ${ }^{11,14}$ Although there are contradictory results about the length of hospital stay in substance addictions, the general belief is that hospitalization durations are shorter for substance addiction than for other psychiatric disorders. ${ }^{7,15,16}$ Reasons for this shorter hospitalization period are the younger age of addicted patients, the low prevalence of medical comorbidities, and the high rate of discharge after detoxification among these patients. More research is needed on the duration of hospitalization in psychiatric illnesses, which are still uncertain and influenced by many variables.

\section{Objective}

The current study purposed to investigate the duration of hospitalization of patients admitted to the Gazi University Hospital Psychiatry Unit in Turkey between 2005 and 2016 and the factors affecting this period.

\section{Methods}

Gazi University Faculty of Medicine is a long-established institution in Ankara providing both educational and health services since 1976. Its inpatient unit has a bed capacity for 15 male patients and 16 female patients. There is a separate 12-bed inpatient treatment unit for the treatment of alcohol and substance addiction. Gazi University Hospital Psychiatry Clinic provides inpatient services to between 300 and 500 people per year. This number varies according to the capacity of the inpatient service. In this study, the hospitalization records of all patients hospitalized in the Psychiatry Department of Gazi University Hospital between January 1, 2005 and December 31, 2016 were evaluated.

This research is a retrospective descriptive study. Data was retrieved from computer records after the necessary permits were received from hospital management.

Axis 1 and 2 diagnoses of patients were evaluated by psychiatrists according to The Diagnostic and Statistical Manual of Mental Disorders, Fourth Edition, Text Revision (DSM IV-TR). Because of the need for a universal evaluation of all disease groups, ICD-10 diagnostic classification is used in the hospital database. For this reason, the diagnosis of evaluated patients was recorded in the system with the most suitable ICD-code. Diagnostic evaluations of patients are addressed in a very comprehensive and detailed manner in this unit. Patients were evaluated by research assistants, senior associate assistants, and in general visits.

In this study the psychotic disorders (schizophrenia, delusional disorders, etc.) and the diseases coded with psychotic symptoms in ICD-10 were classified as "Has psychotic symptoms." Patients with other psychiatric diseases in addition to their primary psychiatric diagnosis were classified as "Has comorbid psychiatric disease." Comorbid physical diseases were handled similarly. First, hospitalization data was used for patients who had recurrent admissions, limiting the effect of identical patient data on the results.

From hospital records, data for 7027 admissions was obtained for a 12-year period. The data showed that 76 people were discharged on their hospitalization day, 55 people had non-psychiatric primary diagnoses (hypertension, pulmonary embolism, ischemic heart disease, etc), 163 people were followed-up for a single day, and 34 patients had missing data; therefore, these groups were not included in the analysis. Among the remaining 6699 cases, 1570 were recurrent admissions, which were also excluded from the analysis. As a result, the data for 5129 admissions was included in the analysis.

\subsection{Data Analysis}

Statistical Package for Social Sciences (SPSS) for Windows 23.0 was used for the analysis of data. Mean standard deviation, median, frequency, and percentage values were presented for descriptive statistics. The chi-square test was used to compare qualitative data, and Fisher exact test was used in necessary cases. The Kolmogorov-Smirnov test was used to investigate whether the data fit with normal distribution. Mann-Whitney U test and Kruskal-Wallis test were used to compare groups in the sample that did not comply with normal distribution. Logistic regression analysis was used to determine the predictors of the duration of hospital stay. A hospitalization period of 28 days or less was accepted as short-term hospitalization. This limit was determined according to the average length of hospital stay $(28.66 \pm 17.25)$ in the sample. The significance value was based on 0.05 in the study.

\section{Results}

Between 2005 and 2016, 5129 patients were treated in the Gazi University Hospital Psychiatry Unit with 6699 admissions. Each patient was hospitalized 1.30 times on average.

The evaluation of the hospitalization period according to patient demographic and disease characteristics is demonstrated in Table 1 . The mean age of patients was $45.27 \pm 14.69($ median $=44.00)$. Males comprised $62.5 \%$ of the sample $(n=3204)$, while $6.7 \%(n=346)$ had a physical comorbidity and $10.8 \%$ had $(n=552)$ a psychiatric comorbidity. Female gender, psychiatric comorbidity, psychotic symptoms, and recurrent admissions were 
Table 1. Evaluation of Hospitalization Period According to Demographic and Disease Characteristics of Patients $(n=5129)$

\begin{tabular}{|c|c|c|c|c|c|}
\hline Variables & & No. $(\%)$ & Mean \pm SD & Median & $\boldsymbol{P}$ \\
\hline \multirow{6}{*}{ Age } & $18-25$ & $400(7.8)$ & $20.57 \pm 13.55$ & 18.00 & \multirow{6}{*}{$<0.001^{\mathrm{a}}$} \\
\hline & $26-35$ & 1124 (21.9) & $24.01 \pm 17.17$ & 22.00 & \\
\hline & $36-45$ & 1209 (23.6) & $29.49 \pm 16.68$ & 28.00 & \\
\hline & $46-55$ & $1084(21.1)$ & $30.74 \pm 16.91$ & 29.00 & \\
\hline & $56-65$ & $824(16.1)$ & $33.06 \pm 17.40$ & 33.00 & \\
\hline & $\geq 66$ & $488(9.5)$ & $31.08 \pm 17.48$ & 31.00 & \\
\hline \multirow{2}{*}{ Gender } & Female & $1925(37.5)$ & $30.34 \pm 17.46$ & 29.00 & \multirow{2}{*}{$<0.001^{\mathrm{b}}$} \\
\hline & Male & $3204(62.5)$ & $27.65 \pm 17.04$ & 26.00 & \\
\hline \multirow{2}{*}{ Physical comorbidity } & No & $4783(93.3)$ & $28.55 \pm 17.30$ & 27.00 & \multirow{2}{*}{$0.059^{b}$} \\
\hline & Yes & $346(6.7)$ & $30.16 \pm 16.36$ & 28.00 & \\
\hline \multirow{2}{*}{ Psychiatric comorbidity } & No & 4577 (89.2) & $28.22 \pm 17.19$ & 27.00 & \multirow{2}{*}{$<0.001^{\mathrm{b}}$} \\
\hline & Yes & $552(10.8)$ & $32.31 \pm 17.33$ & 31.00 & \\
\hline \multirow{2}{*}{ Psychotic symptoms } & No & $3459(67.4)$ & $26.92 \pm 16.99$ & 26.00 & \multirow{2}{*}{$<0.001^{\mathrm{b}}$} \\
\hline & Yes & $1670(32.6)$ & $32.27 \pm 17.12$ & 30.00 & \\
\hline \multirow{3}{*}{ Hospitalization type } & Normal & $4989(97.3)$ & $28.63 \pm 17.25$ & 27.00 & \multirow{3}{*}{$0.650^{\mathrm{a}}$} \\
\hline & Immediate & $114(2.2)$ & $29.42 \pm 17.35$ & 28.00 & \\
\hline & Forensic & $26(0.5)$ & $30.42 \pm 16.54$ & 29.00 & \\
\hline \multirow{2}{*}{ Admission type } & Single admission & 4085 (79.6) & $27.86 \pm 17.01$ & 26.00 & \multirow{2}{*}{$<0.001^{\text {b }}$} \\
\hline & Recurrent admissions & $1044(20.4)$ & $31.77 \pm 17.82$ & 31.00 & \\
\hline \multirow{4}{*}{ Admission season } & Winter & $1258(24.5)$ & $29.68 \pm 17.41$ & 28.00 & \multirow{4}{*}{$<0.001^{\mathrm{a}}$} \\
\hline & Spring & $1325(25.8)$ & $28.60 \pm 17.09$ & 28.00 & \\
\hline & Summer & $1321(25.8)$ & $26.58 \pm 16.81$ & 25.00 & \\
\hline & Autumn & $1225(23.9)$ & $29.92 \pm 17.51$ & 28.00 & \\
\hline
\end{tabular}

SD: Standard deviation.

${ }^{\mathrm{a}}$ Kruskal-Wallis. ${ }^{\mathrm{b}}$ Mann Whitney $\mathrm{U}$ test.

determined to increase the hospitalization period significantly (Table 1).

Depressive disorder was the most common psychiatric disorder among patients admitted to the Psychiatry Department $(n=1063,20.73 \%)$. This was followed by schizophrenia, alcohol addiction, and substance addiction (16.75\%, $13.80 \%$, and $12.15 \%$, respectively). The distribution of these disorders is shown in Figure 1. The duration of hospitalization and the average age of the patients with these psychiatric disorders are shown in
Figure 2. Eating disorder (ED) was the psychiatric disorder with the longest duration of hospitalization (35.96 \pm 21.65$)$, while substance addiction had the shortest duration $(17.02 \pm 12.01)$. The youngest patients were substance addicts $(30.73 \pm 8.03)$, while the oldest patients were those with recurrent depressive disorder $(54.92 \pm 11.92)$.

Distribution of the duration of hospitalization according to the years is shown in Table 2. This period between 2005 and 2009 was longer than 30 days. While the longest was observed between these years, the shortest hospitalization

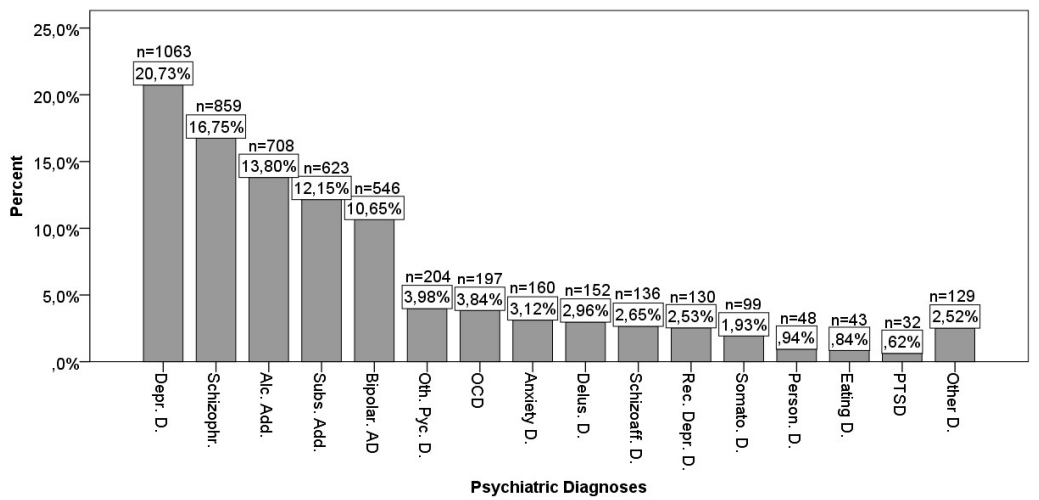

Figure 1. Distribution of Psychiatric Diagnoses.

Depr. D.: Depressive Disorder, Schizoph.: Schizophrenia, Alc. Add.: Alcohol Addiction, Subs. Add.: Substance Addiction, Bipolar AD.: Bipolar Affective Disorder, Oth. Psy. D.: Other Psychiatric Disorder, OCD: Obsessive-Compulsive Disorder, Anxiety D.: Anxiety Disorder, Delus. D.: Delusional Disorder, Schizoaff. D.: Schizoaffective Disorder, Rec. Depr. D.: Recurrent Depressive Disorder, Somato. D.: Somatoform Disorder, Person. D.: Personality Disorder, Eating D.: Eating Disorder, PTSD: Post-Traumatic Stress Disorder, Other D.: Other Disorder 


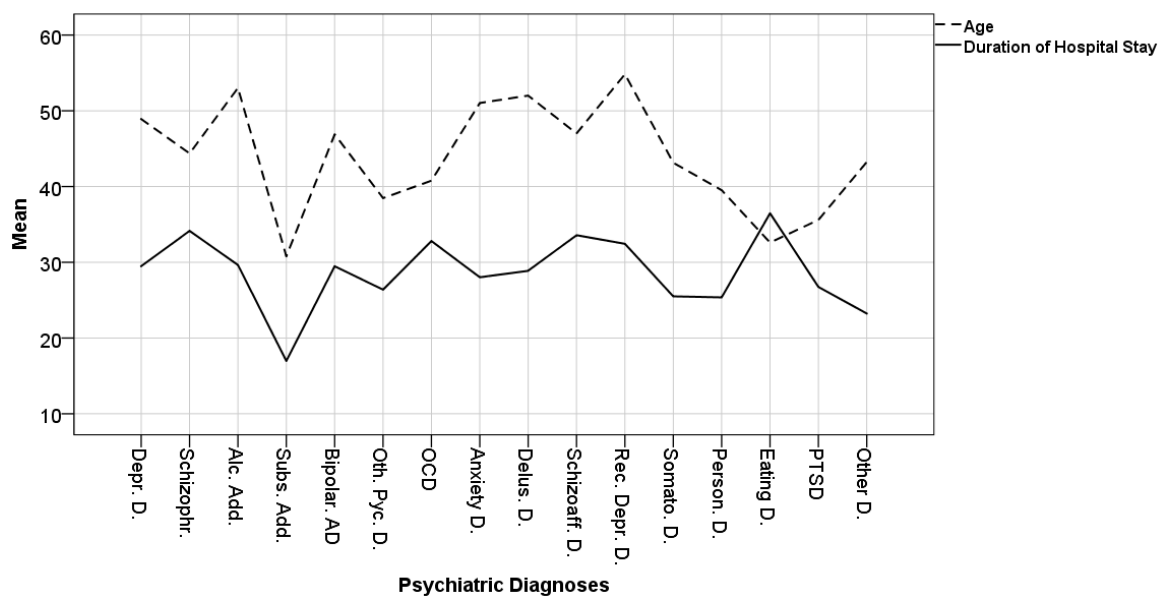

Figure 2. Mean Hospitalization Period and Age According to Psychiatric Diagnoses.

Depr. D.: Depressive Disorder, Schizoph.: Schizophrenia, Alc. Add.: Alcohol Addiction, Subs. Add.: Substance Addiction, Bipolar AD.: Bipolar Affective Disorder, Oth. Psy. D.: Other Psychiatric Disorder, OCD: Obsessive-Compulsive Disorder, Anxiety D.: Anxiety Disorder, Delus. D.: Delusional Disorder, Schizoaff. D.: Schizoaffective Disorder, Rec. Depr. D.: Recurrent Depressive Disorder, Somato. D.: Somatoform Disorder, Person. D.: Personality Disorder, Eating D.: Eating Disorder, PTSD: Post-Traumatic Stress Disorder, Other D.: Other Disorder.

period was between 2013 and 2016. This difference was found to be significant $\left(\chi^{2}=536.475 ; P<0.001\right)$.

The results of the logistic regression analysis for hospitalization duration is shown in Table 3. In this analysis, the length of hospital stay was taken as the dependent (predicted) variable. The value that distinguishes shortterm and long-term hospitalization was considered as 28 days, because this value was the average hospitalization period in the sample (Table 2). Patients aged 26 and over had hospitalizations 1.5- to 3.6-fold longer than those between the ages of 18-25 (Table 3). Women had a 1.15fold (odds ratio $[\mathrm{OR}]=0.868,95 \%$ CI for $\mathrm{OR}=0.765$ 0.986) longer hospitalization period than men. Similarly, the initial hospitalization periods of patients with recurrent admissions were 1.5 -fold $(\mathrm{OR}=1.541,95 \% \mathrm{CI}$ for $\mathrm{OR}=1.333-1.783$ ) longer than those of patients with a single admission. Schizophrenia, schizoaffective disorder, depressive disorder, recurrent depressive disorder, alcohol addiction, obsessive compulsive disorder (OCD), and ED were found to be psychiatric disorders that prolonged the duration of hospitalization (OR varied between 1.5 and 2.8). The only variable that reduced this period was diagnosis of substance addiction $(\mathrm{OR}=0.537,95 \% \mathrm{CI}$ for $\mathrm{OR}=0.392-0.736$ ).

\section{Discussion}

This study investigated the demographic variables, diagnoses, disease characteristics, and other possible factors that may affect the duration of hospitalization. The most frequently hospitalized psychiatric patients had diagnoses of depressive disorder, schizophrenia, and alcohol addiction $(20.73 \%, 16.75 \%$, and $13.80 \%$, respectively). All of these diseases extended the length of the hospitalization period (Table 3 ). The fourth most frequently hospitalized group was substance addicted patients (Figure 1). The diagnosis of a substance addiction was the only psychiatric disorder that shortened the length of hospitalization period (Table 3). Mean hospitalization

Table 2. Distribution of Hospital Stay According to Years $(n=5129)$

\begin{tabular}{|c|c|c|c|c|c|}
\hline Hospitalization Year & $\mathbf{n}$ & Mean \pm SD & $95 \% \mathrm{Cl}$ for Mean & Min-Max & Median \\
\hline 2005 & 249 & $35.05 \pm 17.20$ & $32.91-37.20$ & $1-99$ & 34.00 \\
\hline 2006 & 488 & $36.15 \pm 20.95$ & $34.29-38.01$ & $1-110$ & 35.00 \\
\hline 2007 & 412 & $36.94 \pm 19.86$ & $35.02-38.86$ & $1-114$ & 36.00 \\
\hline 2008 & 458 & $36.43 \pm 19.93$ & $34.60-38.26$ & $1-119$ & 36.00 \\
\hline 2009 & 447 & $31.19 \pm 16.03$ & $29.70-32.69$ & $1-90$ & 31.00 \\
\hline 2010 & 498 & $27.25 \pm 14.85$ & $25.95-28.56$ & $1-108$ & 27.00 \\
\hline 2011 & 471 & $26.34 \pm 14.54$ & $25.02-27.66$ & $1-91$ & 26.00 \\
\hline 2012 & 471 & $26.12 \pm 14.07$ & $24.85-27.40$ & $1-74$ & 26.00 \\
\hline 2013 & 543 & $22.45 \pm 13.23$ & $21.33-23.57$ & $1-91$ & 22.00 \\
\hline 2014 & 463 & $20.41 \pm 14.28$ & $19.11-21.72$ & $1-81$ & 18.00 \\
\hline 2015 & 315 & $24.02 \pm 13.87$ & $22.48-25.56$ & $1-69$ & 23.00 \\
\hline 2016 & 314 & $23.22 \pm 13.50$ & $21.72-24.72$ & $1-83$ & 22.00 \\
\hline Total & 5129 & $28.66 \pm 17.25$ & $28.19-29.13$ & $1-119$ & 27.00 \\
\hline
\end{tabular}

$\chi^{2}=536.475 ; d f=11 ; P<0.001$

SD: Standard deviation; $\mathrm{Cl}$ : Confidence Interval; Min: Minimum; Max: Maximum. 
Table 3. Evaluation of Hospitalization Period With Logistic Regression Analysis ( $n=5129)$

\begin{tabular}{|c|c|c|c|c|c|c|}
\hline \multirow{2}{*}{ Variables } & & \multicolumn{2}{|c|}{ Length of Hospital Stay } & \multicolumn{3}{|c|}{ Statistical Analysis } \\
\hline & & $\leq 28$ Days $(n=2706)$ & $>28$ Days $(n=2423)$ & Whald 20 & $\boldsymbol{p}$ & 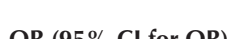 \\
\hline \multirow{6}{*}{ Age } & 1825 & 300 & & & & \\
\hline & $26-35$ & $743(27.5)$ & $381(15.7)$ & 9.364 & 0.002 & $1.539(1.168-2.029)$ \\
\hline & $36-45$ & $608(22.5)$ & $601(24.8)$ & 35.238 & $<0.001$ & $2.307(1.751-3.041)$ \\
\hline & $46-55$ & $510(18.8)$ & $574(23.7)$ & 40.292 & $<0.001$ & $2.501(1.884-3.319)$ \\
\hline & $56-65$ & $323(11.9)$ & $501(20.7)$ & 74.611 & $<0.001$ & 3.668 (2.731-4.926) \\
\hline & $\geq 66$ & $213(7.9)$ & $275(11.3)$ & 47.864 & $<0.001$ & $3.055(2.227-4.192)$ \\
\hline \multirow{2}{*}{ Gender } & Female & $940(34.7)$ & $985(40.7)$ & & & 1.000 \\
\hline & Male & $1766(65.3)$ & $1438(59.3)$ & 4.747 & 0.029 & $0.868(0.765-0.986)$ \\
\hline \multirow{2}{*}{ Admission type } & Single admission & $2240(82.8)$ & $1845(76.1)$ & & & 1.000 \\
\hline & Recurrent admission & $466(17.2)$ & $578(23.9)$ & 34.067 & $<0.001$ & $1.541(1.333-1.783)$ \\
\hline Physical comorbidity* & Yes & $174(6.4)$ & $172(7.1)$ & 0.361 & 0.548 & $0.932(0.742-1.171)$ \\
\hline Psychiatric comorbidity* & Yes & $229(8.5)$ & $323(13.3)$ & 28.412 & $<0.001$ & $1.683(1.390-2.038)$ \\
\hline Schizophrenia* & Yes & $363(13.4)$ & $496(20.5)$ & 36.139 & $<0.001$ & $2.286(1.746-2.993)$ \\
\hline Delusional disorder* & Yes & $77(2.8)$ & $75(3.1)$ & 2.155 & 0.142 & $1.350(0.904-2.014)$ \\
\hline Schizoaffective disorders* & Yes & $57(2.1)$ & $79(3.3)$ & 12.695 & $<0.001$ & $2.139(1.408-3.249)$ \\
\hline Bipolar Affective disorder* & Yes & $295(10.9)$ & $251(10.4)$ & 2.898 & 0.089 & $1.285(0.963-1.715)$ \\
\hline Depressive disorder* & Yes & $534(19.7)$ & $529(21.8)$ & 10.377 & 0.001 & $1.540(1.184-2.003)$ \\
\hline Recurrent depressive disorder* & Yes & $46(1.7)$ & $84(3.5)$ & 10.519 & 0.001 & $2.069(1.333-3.211)$ \\
\hline Anxiety disorder* & Yes & $81(3.0)$ & $79(3.3)$ & 3.055 & 0.080 & $1.420(0.958-2.103)$ \\
\hline Somatoform disorder* & Yes & $57(2.1)$ & $42(1.7)$ & 0.616 & 0.433 & $1.207(0.754-1.931)$ \\
\hline Alcohol addiction* & Yes & $323(11.9)$ & $385(15.9)$ & 12.558 & $<0.001$ & $1.669(1.257-2.216)$ \\
\hline Substance addiction* & Yes & $508(18.8)$ & $115(4.7)$ & 14.922 & $<0.001$ & $0.537(0.392-0.736)$ \\
\hline Post-traumatic stress disorder* & Yes & $18(0.7)$ & $14(0.6)$ & 1.291 & 0.256 & $1.547(0.729-3.282)$ \\
\hline Obsessive compulsive disorder* & Yes & 78 (2.9) & $118(4.9)$ & 27.781 & $<0.001$ & $2.718(1.874-3.943)$ \\
\hline Eating disorders* & Yes & $19(0.7)$ & $24(1.0)$ & 9.377 & 0.002 & $2.825(1.453-5.493)$ \\
\hline Personality disorders* & Yes & $33(1.2)$ & $15(0.6)$ & 0.189 & 0.664 & $0.864(0.447-1.672)$ \\
\hline
\end{tabular}

$X^{2}=532.850 ; P<0.001 ;$ Nagelkerke $R^{2}=0.132$

$\mathrm{OR}$, odds ratio; $\mathrm{Cl}$, confidence interval.

$*$ The reference value for these variables is the absence of disease $(\mathrm{No}=0$ [Reference], Yes $=1)$.

period was found to be $28.66 \pm 17.25$ days in this study. The length of hospitalization was shown to decrease within the years (Table 2). In addition, female gender, presence of psychiatric comorbidity, and older age were other variables that prolonged hospital stay (Table 3).

While there is no standard period of treatment in psychiatric disorders, the duration of hospitalization showed significant differences according to the disease and the patient. In studies investigating short-term and long-term hospitalizations, both hospitalization types were shown to have possible advantages. ${ }^{17}$ It is been reported that significantly greater improvement is seen in areas such as cognitive functions, self-esteem, and interpersonal relations in short-term hospitalizations. Moreover, it was determined that there was a more significant recovery rate in the area of the most significant psychopathology in long-term hospitalizations. Researchers have claimed that the therapeutic relationship had importance in this regard. ${ }^{5}$ Data obtained from other studies should be evaluated in this context. It is remarkable that the length of hospitalization period gradually decreased in the institution under study. The longest hospitalization period was observed between 2005 and 2008, while the shortest hospitalization period was seen between 2013 and 2016 (Table 2). Even if the diagnostic approach and treatment methods were similar, there may be many explanations for this difference. Some of these reasons are low bed availability allocated for psychiatric patients, increased awareness and number of psychiatric disorders, increased accessibility to healthcare services, economic costs imposed by long-term hospitalization, and the health policies established to decrease these costs. It is reported that the length of hospitalization in both public and private hospitals has been reduced with the Health Transformation Program policies that came into force in 2003 in Turkey. ${ }^{18}$ In addition to shortening the length of hospital stay, the almost doubled bed turnover rate prevented healthcare staff from being able to meet the increased workload. ${ }^{18}$ While decreased length of hospitalization period lowers the cost directly, reasons such as possible repetition of the disease due to reduced service quality, re-hospitalizations, and low working efficiency impose indirect financial burdens. ${ }^{19} \mathrm{~A}$ study that examined the relationship between length of hospital stay and recurrent admissions revealed that a reduction in hospitalization period increased readmission rates. ${ }^{20}$ The researchers stated that the financial profit obtained by the shortened hospital stay was below the financial loss caused by recurrent admissions. For this reason, applied health policies should practice an understanding that provides balance in this issue. 
It has been shown that length of hospitalization is affected by some demographic variables, ${ }^{5-7}$ among which, age and gender are the most important variables. While conflicting results on gender have been obtained from various studies, it was demonstrated in a comprehensive study performed in the United States that female patients were hospitalized for a longer period. In the current study, the length of hospitalization period was 1.15-fold longer in females than in males. Similarly, advancing age was found to be another variable that prolonged hospitalization period (Table 3 ). It has been reported that adverse drug reactions, comorbidities, sleep disorders, and cognitive disorders, which are observed more often with increased age, also prolonged hospitalization period. ${ }^{5,21}$ Some studies have stated that the age variable showed a bimodal distribution in predicting hospitalization period. ${ }^{5}$

The hospital under study has a separate unit for treating alcohol and substance addiction. This unit is a treatment center for those primarily diagnosed with such diseases. The first target in the treatment of alcohol and substance addiction is detoxification. After completing this basic treatment program, a discharge plan is prepared to increase the adaptation of such patients to social life. After discharge, treatment continues with group therapy. In other words, treatment takes a long time, but the length of hospitalization seems short. This may be the reason why the presence of substance addiction was determined as a variable that shortened the length of hospitalization in the current study. Hospitalization period was clearly longer in the diagnosis of alcohol addiction than in the diagnosis of substance addiction (Figure 2). Even, this average was above the sample average. Therefore, the diagnosis of alcohol addiction was found to increase the hospitalization period 1.6-fold (Table 3). Different studies in the literature reported on the effect of alcohol addiction on the duration of hospitalization. If alcohol and substance addictions are secondary to psychiatric disorders, it has been suggested that the length of hospitalization is longer. ${ }^{11,22}$ For this reason, whether these diseases are the primary diagnosis or comorbidities should be determined while evaluating the length of hospitalization for alcohol and substance addictions.

Psychotic disorders and affective disorders make up the majority of hospitalizations in inpatient treatment in psychiatry. ${ }^{23,24}$ In the current study, depressive disorder accounted for $20 \%$ of hospitalizations, while schizophrenia accounted for $16 \%$ (Figure 1). However, the hospitalization period in these disorders was 1.5 and 2.2 times longer, respectively (Table 3 ). Both the frequent and the long-term hospitalizations for these disorders explain the high burden of the diseases. A 2018 study examined 1479 patients hospitalized in a psychiatric unit. ${ }^{7}$ The results indicated that schizophrenia, affective disorders, advanced age, and recurrent admissions were associated with a long hospital stay. The presence of similar results in the current study indicates that these variables are important predictors of the duration of hospitalization.
While ED and OCD are evaluated as different diagnostic categories, they show similarities with regard to high comorbidity rates, clinical characteristics, and personality traits. Although obsessive and perfectionist personality traits provide an inclination to both disorders, the presence of OCD symptoms is a risk factor for ED. ${ }^{25}$ The fact that cognitive behavior therapy (CBT) is used to treat both disorders is another common characteristic. Studies have shown that OCD and ED are associated with long hospitalization periods. The use of CBT in these disorders and a high rate of psychiatric comorbidity have been reported as variables that increased hospitalization period. ${ }^{26}$ Similar to the information in the literature, longterm (6-8 weeks) CBT is applied for most of these patients in the inpatient unit under study. For this reason, the hospitalization period for these patients could have seemed longer compared with patients with other disorders. ED is also the diagnostic group with the longest hospitalization period in the current study (Figure 2). In addition to treatment selection and psychiatric comorbidity variables, accompanying serious medical problems such as hypocalcemia and hypotension in patients with an ED are further reasons for prolonged hospital stay.

Although the current study was performed in a longestablished institution (the Gazi University Faculty of Medicine), there were limitations. This study was a singlecenter study, and comorbidities were not examined in detail. There was a lack of complete demographic data, and drug treatments were not specified. Lack of evaluation in subgroups of diseases can also be considered as a limitation. For example, in bipolar disorder, the duration of hospitalization may be different during periods of mania than in periods of depression. Subgroups were not analyzed in the current study, because the study aimed to determine a general framework that would cover the entire sample. Comparing the lengths of hospitalization in these subgroups may be the target of further studies.

\section{Conclusion}

Schizophrenia and depression are the most common psychiatric disorders in the inpatient service, and these disorders prolong hospitalization periods. This period is considerably less in substance addiction than in other psychiatric disorders. In future studies on hospitalization periods in alcohol and substance addictions, variables such as the status of the diagnosis of addiction (primary, secondary), consumed doses of alcohol/substance, and environmental conditions (job, economic situation, etc) should be addressed. Non-clinical variables such as year of hospitalization may affect the length of hospital stay. Many factors such as treatment modalities, health policies, etc can play a role in this. Future studies should investigate whether shortened hospitalization periods increase recurrent admissions.

\section{Authors' Contributions}

Study design: BG, BC; Data collection and/or processing: 


\section{Research Highlights}

\section{What Is Already Known?}

Long-term hospitalization is a serious financial burden in psychiatric diseases. Schizophrenia and mood disorders are important diseases with a long duration of hospitalization.

\section{What This Study Adds?}

Non-clinical variables (e.g., hospitalization year) may affect the duration of hospitalization in psychiatric disorders. In psychiatric disorders, the duration of hospitalization decreases with years. Schizophrenia and depression are the two most important psychiatric disorders with long hospitalization periods. The duration of hospitalization is shorter in substance use disorders than in other psychiatric disorders.

BG; Manuscript writing: BG, BC; Statistical analysis: BG; Literature review and critical revision: $\mathrm{BG}, \mathrm{BC}$.

\section{Conflict of Interest Disclosures}

The authors declare that they have no conflicts of interest.

\section{Ethical Approval}

Authorization for this study was obtained from the Ethics Committee of Gazi University. Because hospital records were used in the study, permission was obtained from hospital management.

\section{References}

1. World Health Organization (WHO). Mental Health Action Plan 2013-2020. Geneva: WHO; 2013.

2. Steel Z, Marnane C, Iranpour C, et al. The global prevalence of common mental disorders: a systematic review and metaanalysis 1980-2013. Int J Epidemiol. 2014;43(2):476-493. doi:10.1093/ije/dyu038.

3. Vigo D, Thornicroft G, Atun R. Estimating the true global burden of mental illness. Lancet Psychiatry. 2016;3(2):171-178. doi:10.1016/s2215-0366(15)00505-2.

4. Henderson C, Noblett J, Parke H, et al. Mental healthrelated stigma in health care and mental health-care settings. Lancet Psychiatry. 2014;1(6):467-482. doi:10.1016/s22150366(14)00023-6.

5. Gopalakrishna G, Ithman M, Malwitz K. Predictors of length of stay in a psychiatric hospital. Int J Psychiatry Clin Pract. 2015;19(4):238-244. doi:10.3109/13651501.2015.1062522.

6. Bolden L, Wicks MN. Length of stay, admission types, psychiatric diagnoses, and the implications of stigma in African Americans in the nationwide inpatient sample. Issues Ment Health Nurs. 2005;26(10):1043-1059. doi:10.1080/01612840500280703.

7. Habermeyer B, De Gennaro H, Frizi RC, Roser P, Stulz N. Factors associated with length of stay in a Swiss mental hospital. Psychiatr Q. 2018;89(3):667-674. doi:10.1007/s11126-0189569-4.

8. World Health Organization (WHO). World Drug Report 2018. Vienna: United Nations Publications; 2018.

9. World Health Organization (WHO). Global Status Report on
Alcohol and Health 2018. Geneva: WHO; 2018.

10. Regier DA, Farmer ME, Rae DS, etal.Comorbidity of mental disorders with alcohol and other drug abuse: results from the Epidemiologic Catchment Area (ECA) study. JAMA. 1990;264(19):2511-2518. doi:10.1001/jama.1990.03450190043026.

11. Gerding LB, Labbate LA, Measom MO, Santos AB, Arana GW. Alcohol dependence and hospitalization in schizophrenia Schizophr Res. 1999;38(1):71-75. doi:10.1016/s09209964(98)00177-7.

12. Gottheil E, McLellan AT, Druley KA. Length of stay, patient severity and treatment outcome: sample data from the field of alcoholism. J Stud Alcohol. 1992;53(1):69-75. doi:10.15288/ jsa.1992.53.69.

13. Schatzberg AF, Weiss RD, Brady KT, Culpepper L. Bridging the clinical gap: managing patients with co-occurring mood anxiety, and alcohol use disorders. Introduction. CNS Spectr. 2008:13(4 Suppl 6):3.

14. Botha UA, Koen L, Joska JA, et al. The revolving door phenomenon in psychiatry: comparing low-frequency and highfrequency users of psychiatric inpatient services in a developing country. Soc Psychiatry Psychiatr Epidemiol. 2010;45(4):461468. doi:10.1007/s00127-009-0085-6.

15. Pauselli L, Verdolini N, Bernardini F, Compton MT, Quartesan R. Predictors of length of stay in an inpatient psychiatric unit of a general hospital in Perugia, Italy. Psychiatr Q. 2017:88(1):129140. doi:10.1007/s11126-016-9440-4.

16. Wolff J, McCrone P, Patel A, Kaier K, Normann C. Predictors of length of stay in psychiatry: analyses of electronic medical records. BMC Psychiatry. 2015;15:238. doi:10.1186/s12888015-0623-6

17. Slade M, Byford S, Barrett B, et al. Alternatives to standard acute in-patient care in England: short-term clinical outcomes and cost-effectiveness. Br J Psychiatry Suppl. 2010;53:s14-19. doi:10.1192/bjp.bp.110.081059

18. Kasapoğlu A. Transformation of health policies in Turkey. Journal of Sociological Research (JSR). 2016;19(2):131-174. doi:10.18490/sosars. 261673

19. Koçak C, Illhan MN, Kuruoğlu A. Evaluation of treatment costs invoiced to the social security institution for inpatients hospitalized in psychiatric ward of Gazi university hospital. Clin Psy. 2017:20(4):268-275. doi:10.5505/kpd.2017.66588.

20. Wickizer TM, Lessler D. Do treatment restrictions imposed by utilization management increase the likelihood of readmission for psychiatric patients? Med Care. 1998;36(6):844-850. doi:10.1097/00005650-199806000-00008.

21. Classen DC, Pestotnik SL, Evans RS, Lloyd JF, Burke JP. Adverse drug events in hospitalized patients: excess length of stay, extra costs, and attributable mortality. JAMA. 1997;277(4):301-306. doi:10.1001/jama.1997.03540280039031.

22. Tekin Uludağ Y, Güleç G. Prevalence of substance use in patients diagnosed with schizophrenia. Noro Psikiyatr Ars. 2016;53(1):4-11. doi:10.5152/npa.2015.8827.

23. Hallak JE, Crippa JA, Vansan G, Zuardi AW. Diagnostic profile of inpatients as a determinant of length of stay in a general hospital psychiatric unit. Braz J Med Biol Res. 2003;36(9):1233-1240. doi:10.1590/s0100-879x2003000900014.

24. Hamilton JE, Passos IC, de Azevedo Cardoso T, et al. Predictors of psychiatric readmission among patients with bipolar disorder at an academic safety-net hospital. Aust N Z J Psychiatry. 2016;50(6):584-593. doi:10.1177/0004867415605171.

25. Karayilan S, Erol A.. Obsessive compulsive disorder and eating disorder. Turkiye Klinikleri J Psychiatry. 2012;5(3):76-84.

26. Simpson HB, Wetterneck CT, Cahill SP, et al. Treatment of obsessive-compulsive disorder complicated by comorbid eating disorders. Cogn Behav Ther. 2013;42(1):64-76. doi:10.1080/16 506073.2012.751124. 